been reported to secrete a tissue type plasminogen activator ${ }^{1}$ so it may be that the abnormal clone present in this patient has switched on this ability to a greater than normal degree. Alternatively, the underlying process may be related to the procoagulant properties of monocytes with a compensatory secondary hyperfibrinolysis. The clinical presentation, however, is one of bleeding, with no evidence of thrombotic damage over several years. An association of high tPA concentrations and low concentrations of PAI has been shown in patients with liver disease, ${ }^{2}$ but investigations have not shown any liver pathology in this case. A lupus inhibitor seems unlikely given the lack of interference in factor assays, the negative Exner test, and absence of any other evidence of autoimmune disease.

Infusion of FFP seems to be the most appropriate approach in the treatment and prophylaxis of bleeding episodes in this patient. The role of tranexamic acid is debatable-the ability to moderate fibrinolysis is useful, but in these circumstances there is a theoretical risk of precipitating disseminated intravascular coagulation. The administration of DDAVP seems to have no effect.

1 Hart PH, Vitti GF, Burgess DR, Singleton DK, Hamilton JA. Human monocytes can produce tissue-type plasminogen activator. J Exp Med 1989;169:1509-14.

2 Booth NA, Anderson JA, Bennett B. Plasminogen activators in alcoholic cirrhosis: demonstration of increased tissue type and urokinase type activator. J Clin Pathol 1984; type and

\title{
Squamous cell carcinoma arising in endometriosis of the ovary
}

\author{
K N Naresh, V K Ahuja, C Rama Rao, G Mukherjee, M K Bhargava
}

\begin{abstract}
Squamous cell carcinoma arising in ovarian endometriosis is extremely rare, but a case is reported in a 62 year old woman. If such an ovarian tumour is discovered evidence of teratoma, Brenner tumour, or endometriosis should be sought. Extensive sampling is mandatory as the developing tumour often overgrows the foci of endometriosis, thus obliterating evidence of its origin.
\end{abstract}

Malignant change. occurs in foci of endometriosis of which the most common site is the ovary. The resulting tumour is often an endometrioid adenocarcinoma. Mixed müllerian tumours, clear cell adenocarcinomas, ${ }^{1}$ and endometrial stromal sarcomas have also been described. The occurrence of squamous cell carcinoma is extremely rare. ${ }^{2}$ Conversely, squamous cell carcinomas of the ovary usually arise in a pre-existing mature teratoma or a Brenner tumour. Primary squamous cell carcinoma and squamous cell carcinoma arising in ovarian endometriosis form an extremely small proportion of cases. ${ }^{3}$

\section{Case report}

A 62 year old post-menopausal woman was referred to the Institute for evaluation of a pelvic mass. Examination showed a 12-14 week old, immobile, right sided mass in the pelvis. Ultrasonography showed that the mass was $10 \times 12 \mathrm{~cm}$ with hydronephrosis on the right side. Laparotomy was performed, showing a $10 \times 10 \mathrm{~cm}$ right ovarian mass, adherent to the posterior pelvic peritoneum over the sacral promontory and pouch of Douglas and the posterior surface of the uterus. The parametrium on the right side had been infiltrated. There was no evidence of tumour elsewhere in the abdominal cavity. A total abdominal hysterectomy, left salpingooophorectomy and debulking of the right ovarian mass with partial omentectomy were carried out. Residual disease was left behind in the parametrium and pouch of Douglas.

After surgery, the patient was given multiagent chemotherapy, but she gradually deteriorated and died two months later. Necropsy was not performed.

\section{Pathology}

Gross examination of the specimen showed a right ovarian mass $10 \mathrm{~cm}$ in diameter infiltrating the posterior wall of the uterine corpus. Its outer surface was nodular and cut section was predominantly white and solid, interspersed with small cystic spaces, $0.3 \mathrm{~cm}-1.5 \mathrm{~cm}$ in dimension. Papillary areas were not identified. The cervix and left adnexae were normal. 


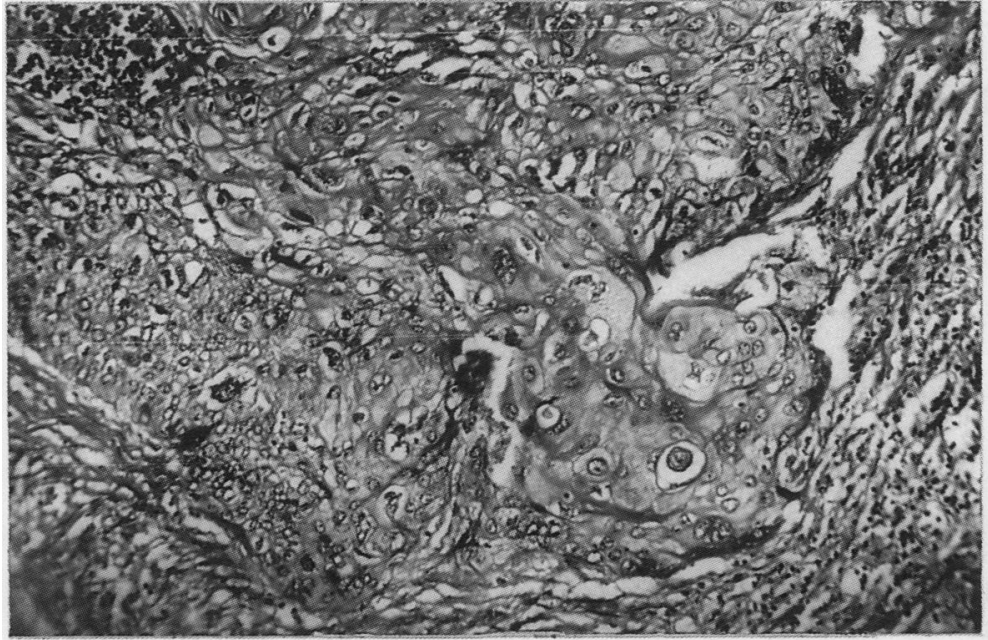

Moderately differentiated squamous cell carcinoma (haematoxylin and eosin).

Histological examination of the ovarian mass showed a malignant epithelial tumour with cells showing intercellular bridges and focal areas of keratinisation (figure). Many areas adjacent to the tumour showed dense areas of collagen with abundant haemosiderin pigmentation. A thorough examination of multiple sections showed small foci of glands lined by columnar epithelium and surrounded by spindle cells. Transition from these benign glandular elements to malignant tumour was not identifiable. Multiple sections from the mass did not show any evidence of teratoma or Brenner tumour. Sections from the uterine body showed that the myometrium had been infiltrated from the serosal aspect. The endometrium, uterine cervix, and left adnexae did not have any evidence of clinical abnormality. A histological diagnosis of squamous cell carcinoma arising in ovarian endometriosis was made.

\section{Discussion}

If squamous carcinoma is found in the ovary, a search for evidence of teratoma, Brenner tumour, or endometriosis in the ovarian tissue adjacent to the tumour needs to be made. To prove that a tumour has arisen from a preexisting focus of endometriosis an attempt should be made to show a transition from benign endometriosis to frankly malignant neoplasm. In most cases, however, the developing tumour overgrows the foci of endometriosis and thus obliterates all evidence of its origin ${ }^{1}$; extensive sampling is therefore mandatory in these cases. An overgrowth of the squamous component in an adenosquamous carcinoma was excluded as the glandular element in our case was benign.

Epidermoid cysts have been described in the ovary. These cysts are usually small (2-14 $\mathrm{mm}$ in diameter) and are incidental findings. Nogales and Silverberg consider that the absence of endometrial, mucinous, and transitional-type epithelium is a prerequisite for a diagnosis of epidermoid cyst and have favoured an origin from ovarian surface epithelium. ${ }^{3}$ Young et al, however, by studying serial sections from the cyst wall, have shown that the squamous epithelium arises as a metaplastic change in the epithelial cell nests, of the type encountered in Brenner tumour. ${ }^{4}$ Malignant change occurring in such a cyst needs to be considered carefully. The tumour under discussion was larger, predominantly solid, and did not show any foci of "Brenner" type epithelium, unlike epidermoid cysts. Furthermore, features which could be clearly attributable to endometriosis (endometrial type glands and fibrotic areas with haemosiderin pigmentation) were seen.

Scully has observed two cases of ovarian squamous cell carcinoma in association with squamous cell carcinoma in situ of the cervix. ${ }^{5}$ In our case, multiple sections from the cervix did not reveal any features of cervical intraepithelial neoplasia. The surface epithelium of the ovary is known to differentiate in an endometrial direction, and it is likely that in this case the squamous cell carcinoma probably arose in a pre-existing benign endometriosis.

A case similar to the one discussed has been described. 6 The squamous carcinoma described in that report arose in an endometriotic cyst. The clinical outcome was similar to that of our case. The patient died three months after surgery with peritoneal deposits and pulmonary and hepatic metastases.

1 Fox H, Buckley CM. Current concepts of endometriosis. Clin Obstet Gynecol 1984;11:279-87.

2 Yetman JJ, Dudzinski MR. Primary squamous cell carcinoma of ovary: A case report and review of literature. Gynecol Oncol 1989;34:240-3.

3 Nogales FF, Silverberg SG. Epidermoid cysts of the ovary. Am J Obstet Gynecol 1976;124:523-8.

4 Young RH, Prat J, Scully RE. Epidermoid cysts of the ovary. Am J Clin Pathol 1980;73:272-6.

5 Scully RE. Tumours of the ovary and maldeveloped gonads. (AFIP Fascicle 16, Second Series). Washington Armed Forces Institute of Pathology, 1979:143-4.

6 Lele SB, Piver MS, Barlow JJ, Tsukada Y. Squamous cell carcinoma arising in ovarian endometriosis. Gynecol Oncol 1977;6:290-3. 